\title{
Analysis of Heat Flows in a Slab Using the Finite Element Model
}

\author{
Amechi Joseph Ujam and Chinagorom Ajike
}

\begin{abstract}
This research work sought to study the pattern of heat flows in a heat conducting clay slab using the finite element model. The theoretical formulation of the finite element model was developed and presented as the general equation governing quasi-harmonic and time dependent field functions. The element equation of the conducting slab under the study was formed and subsequently assembled by taking the summation over all the elements. The assembly equation reduces to a set of linear first order equations which forms the global heat diffusion equation. The findings of the study are presented in the plots which show the solution of the interior mesh points. This is made possible on the application of initial values and boundary conditions that predict completely the time histories of temperature distribution across the isotherms overlaid through the thickness of the slab.
\end{abstract}

Index terms - Element equation, Finite element analysis, Isotherms, Quasi-harmonic.

\section{Introduction}

The crux of this work is to study the heat and mass flows through an isotropic clay slab surface subject to defined boundary conditions. In addition, the heat conducting slab is devoid of external forces i.e. the rate of external heat generation is zero, as well as no accumulation of matter within the material. The geometry of field quantities or continuum may be a problem to a close form solution of field functions encountered in engineering which allows for appropriate algorithm to obtain optimum solutions. Next important is to employ the 'calculus of variation' principle to obtain optimum continuum field functions whose boundary conditions are specified. About all quasi-harmonic phenomena are represented either by the partial derivatives of the function or by the well-known Laplace and Poisson equation, (Ziennkiewicz and Cheung, 1967).

\section{Theoretical Formulation}

In calculus of variation, instead of attempting to locate the points that extremize the function, one or more variables that extremize quantities called functional $(\chi)$, function of the functions that extremize the functional are found. (Ihueze and Ofochebe, 2011) presented the general equation governing quasi-harmonic and time dependent field functions as:

$$
\frac{\partial}{\partial x}\left(k_{x} \frac{\partial \phi}{\partial x}\right)+\frac{\partial}{\partial y}\left(k_{y} \frac{\partial \phi}{\partial y}\right)+\frac{\partial}{\partial z}\left(k_{z} \frac{\partial \phi}{\partial z}\right)+Q-c \frac{\partial \phi}{\partial t}=0 \quad \ldots \ldots
$$

While the Euler's theorem states that if the integral

$$
I(\phi)=\iiint f\left(x, y, z, \phi, \frac{\partial \phi}{\partial x}, \frac{\partial \phi}{\partial y}, \frac{\partial \phi}{\partial z}\right) d x d y d z
$$

is to be minimized, then the necessary and sufficient condition for the minimum to be reached is that the unknown function $\phi(x, y, z)$ should satisfy the following differential equation,

$$
\frac{\partial}{\partial x}\left[\frac{\partial f}{\partial(\partial \phi / \partial x)}\right]+\frac{\partial}{\partial y}\left[\frac{\partial f}{\partial(\partial \phi / \partial y)}\right]+\frac{\partial}{\partial z}\left[\frac{\partial f}{\partial(\partial \phi / \partial z)}\right]-\frac{\partial f}{\partial \phi}=0
$$

within the same region provided that $\phi$ satisfies the same boundary condition in both cases.

From above, it was shown that the equivalent formulation to that of equation (1), is the requirement that the volume integral given below and taken over the whole region, should be minimized.

$$
\chi=\iiint\left[\frac{1}{2}\left\{k_{x}\left(\frac{\partial \phi}{\partial x}\right)^{2}+k_{y}\left(\frac{\partial \phi}{\partial y}\right)^{2}+k_{z}\left(\frac{\partial \phi}{\partial z}\right)^{2}\right\}+\left(Q-c \frac{\partial \phi}{\partial t}\right) \phi\right] d x d y d z(4)
$$


subject to $\phi$ obeying the same boundary condition and however, $\partial \phi / \partial t$ is an invariant.

In a typical case of one-dimensional 'heat and mass' flow through an isotropic clay surface subjected a specific boundary condition and devoid of external force (i.e rate of heat generation $\mathrm{Q}=0$ ), the equivalent functional to be minimized reduces to;

$$
\chi=\int \frac{1}{2}\left[k_{y}\left(\frac{\partial \phi}{\partial y}\right)^{2}-\left(c \frac{\partial \phi}{\partial t}\right) \phi\right] d y
$$

Assuming no accumulation of matter within the sintered clay then $\chi$ can be relaxed for optimization of heat conduction through the thickness of a clay sample. This assumption would be appropriate in a low temperature process displaying free convention without external heat addition.

For the particular case of the steady state heat conduction, the functions, $k_{x}, k_{y}$ and $k_{z}$ may be identified as anisotropic conductivity coefficients, the function $Q$ as the rate of heat generation, the unknown field function as the temperature $(T)$, and $\partial \phi / \partial t$ is due to accumulation of heat at various locations (provided the co-ordinates coincide with the principal axis of the material). The last term of equation (1) can be considered as a prescribed function of position only. Hence equation (5) may be re-written in corresponding heat flow terms as;

$$
\chi=\int \frac{1}{2}\left[k_{y}\left(\frac{\partial T}{\partial y}\right)^{2}-\left(c \frac{\partial T}{\partial t}\right) T\right] d y
$$

The finite element procedure is implemented further by assuming that for the one-dimensional case, heat exchange is executed in a region defined by a straight line (whose length corresponds to the thickness of the of the plate, w) discretized into a finite number of line elements described uniquely by two nodal points, while the nodes correspond to equivalent isotherms overlaid in a regular fashion across the entire surface as illustrated in figure 1 below.

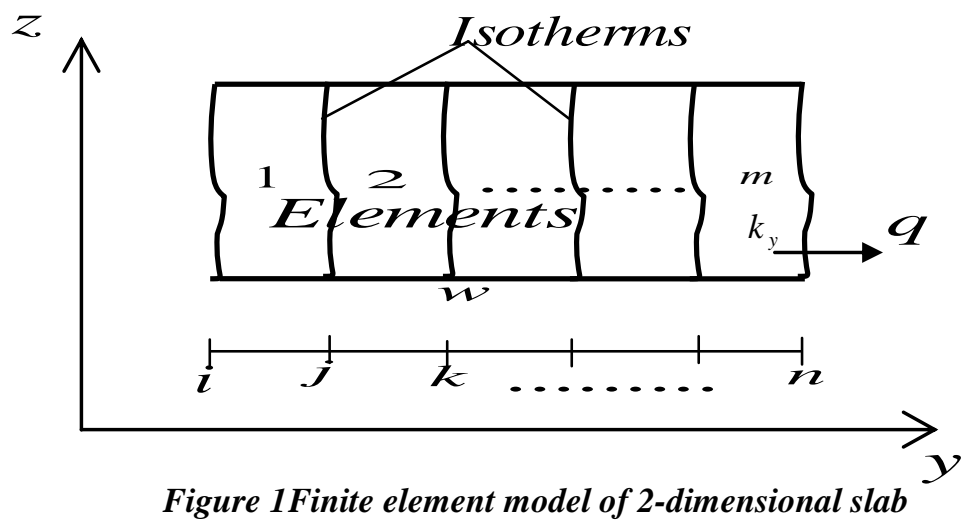

Considering a typical element of the region identified by the 2-nodes in a local co-ordinate system (i, j). In general, if within the element;

Hence;

$$
\{T\}^{e}=\left\{\begin{array}{c}
T_{i} \\
T_{j}
\end{array}\right\} ; N=\left[N_{i} N_{j}\right]
$$

Similarly;

$$
T=[N]\{T\}^{e}=N_{i} T_{i}+N_{j} T_{j}
$$

$$
\frac{\partial T}{\partial t}=[N]\left\{\frac{\partial T}{\partial t}\right\}^{e}
$$

Equation (7) is the shape function and $N$ is the interpolating function.

Where,

$$
N_{i}=\frac{y_{j}-y}{y_{j}-y_{i}}, \quad N_{j}=\frac{y-y_{i}}{y_{j}-y_{i}}
$$


With the nodal values of $T$ now defining uniquely and continuously the function throughout the region the 'functional' $\chi$ can be minimized with respect to these values. This process is best accomplished by evaluating first the contributions to each differential such as $\partial \chi / \partial T_{i}$ from a typical element, then adding all such contributions and equating to zero. Only the elements adjacent to node 1 will contribute to $\partial \chi / \partial T_{i}$ just as only such elements contributed in plane elasticity of the equilibrium equation of such node.

\section{Element Equation Formulation}

If the value of $\chi$ associated with an element is designated with $\chi^{e}$ (implying integration limited to the length of the element) then the next equation is derived by differentiating equation (6);

$$
\frac{\partial \chi^{e}}{\partial T_{i}}=\int\left[k_{y} \frac{\partial T}{\partial y} \frac{\partial}{\partial T_{i}}\left(\frac{\partial T}{\partial y}\right)+c[N] \frac{\partial T}{\partial t} \frac{\partial T}{\partial T_{i}}\right] d y
$$

With $T$ given as the 'shape function' defined by equation (7); evaluating the partial derivatives contained in equation (10) then;

Similarly;

$$
\begin{aligned}
& \frac{\partial \chi^{e}}{\partial T_{i}}=\frac{1}{L^{2}} \int_{y_{i}}^{y_{j}} k_{y}\left(T_{i}-T_{j}\right) d y+\int_{y_{i}}^{y_{j}} c[N]\left\{\frac{\partial T}{\partial t}\right\}^{e} N_{i} d y \\
= & \frac{k_{y}}{L^{2}}\left(T_{i}-T_{j}\right)+\int_{y_{i}}^{y_{j}}\left(c[N] N_{i}\right) d y\left\{\frac{\partial T}{\partial t}\right\}^{e} \quad \ldots \ldots \ldots .(11)
\end{aligned}
$$

$$
\frac{\partial \chi^{e}}{\partial T_{j}}=\frac{k_{y}}{L^{2}}\left(T_{j}-T_{i}\right)+\int_{y_{i}}^{y_{j}}\left(c[N] N_{j}\right) d y\left\{\frac{\partial T}{\partial t}\right\}^{e}
$$

Combining equations (11) and (12) gives a typical element equation of the general form;

$$
\begin{gathered}
\left\{\frac{\partial \chi^{e}}{\partial T}\right\}_{i j}=\frac{k_{y}}{L}\left[\begin{array}{cc}
1 & -1 \\
-1 & 1
\end{array}\right]\left[\begin{array}{l}
T_{i} \\
T_{j}
\end{array}\right]+[p]\left\{\frac{\partial T}{\partial t}\right\}^{e} \quad \ldots \ldots(13) \\
{[p]=p_{i j}=\int_{y_{i}}^{y_{j}} c[N]\left[N^{T}\right] d y=\frac{2 c}{L^{2}}\left[y_{i}{ }^{2} y_{j}+y_{j}^{2} y_{i}+\frac{1}{3}\left(y_{j}^{3}-y_{i}{ }^{3}\right)\right] I}
\end{gathered}
$$

Evidently the first product $[N]\left[N^{T}\right]$ is a scalar, hence the value of $[p]$ in computed by multiplying the value of the integral by unit vector to achieve a balanced degree of freedom with the vector $[h]$.

Hence, the element equation can be expressed in a more concise form as;

$$
\left\{\frac{\partial \chi}{\partial T}\right\}^{e}=h_{i j} T_{j}+p_{i j} \frac{\partial T_{j}}{\partial t}
$$

\section{Assembly Equation}

The final equation of minimization procedure requires the assembly of all the differentials of $\chi$ and equating the result to zero. Typically,

$$
\frac{\partial \chi}{\partial T_{i}}=\sum \frac{\partial \chi^{e}}{\partial T_{i}}=0
$$

the summation being taken over all the elements. Hence in the light of equations (13) and (14) the expression is written as;

Or;

$$
\frac{\partial \chi}{\partial T_{i}}=\sum \sum h_{i j} T_{j}+\sum \sum p_{i j} \frac{\partial T_{j}}{\partial t}=0
$$

$$
\frac{\partial \chi}{\partial T_{i}}=[H]\{T\}+[P]\left\{\frac{\partial T}{\partial t}\right\}=0
$$


In which $[H]$ is the 'stiffness matrix' of the whole assembly, (Astley, 1992) and $[P]$ is a matrix assembled by precisely the same rule as the stiffness matrix from the components of $p_{i j}$.

\section{Final Assembly Equation}

The generation of the final assembly equation for a typical heat conduction surface formulated above may be accomplished in the following synthesized procedures.

Consider a slab of thickness $w=10 \mathrm{~mm}$, thermal conductivity, $k=0.72 \mathrm{~W} / \mathrm{m}^{\circ} \mathrm{C}$, specific heat capacity, $C_{p}$ $=920 \mathrm{~J} / \mathrm{kg}^{\circ} \mathrm{C}$ and density $\rho=1780 \mathrm{~kg} / \mathrm{m}^{3}$ that is initially at a temperature of $30^{\circ} \mathrm{C}$. Say at time $t=0$, one side of the slab is brought in contact with water at $T_{w}=40^{\circ} \mathrm{C}$ at all times, while the other side is subjected to convection to the environment at $T_{\infty}=30^{\circ} \mathrm{C}$, discretized into five (5) similar elements of length $\boldsymbol{L}$ composed of a total of six (6) nodal points. The co-ordinates of these points in the universal system may be described as shown in figure 5.2.

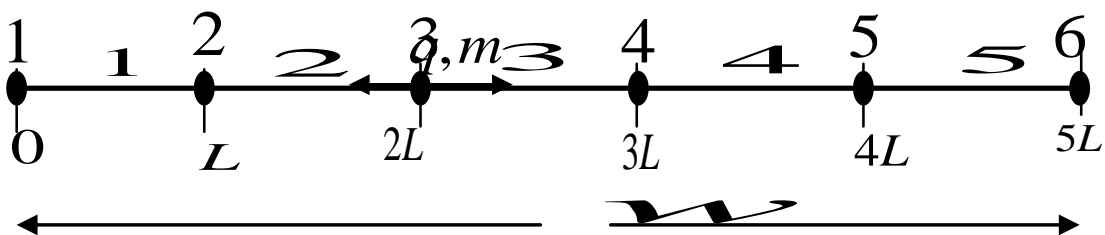

Figure 2Finite element model of a sintered clay slab subjected to heat exchange with the surrounding.

$p_{i j}$ and $h_{i j}$ are evaluated separately for every individual element in global coordinate system. and the assembly obeyed consistent element topology and the resulting minimization equation of the global system is expressed in matrix form as follows:

$$
\frac{k_{y}}{L}\left[\begin{array}{cccccc}
1 & -1 & 0 & 0 & 0 & 0 \\
-1 & 2 & -1 & 0 & 0 & 0 \\
0 & -1 & 2 & -1 & 0 & 0 \\
0 & 0 & -1 & 2 & -1 & 0 \\
0 & 0 & 0 & -1 & 2 & -1 \\
0 & 0 & 0 & 0 & -1 & 1
\end{array}\right]\left[\begin{array}{c}
T_{1} \\
T_{2} \\
T_{3} \\
T_{4} \\
T_{5} \\
T_{6}
\end{array}\right]-c L\left[\begin{array}{cccccc}
2 / 3 & 0 & 0 & 0 & 0 & 0 \\
0 & 4 / 3 & 0 & 0 & 0 & 0 \\
0 & 0 & 4 / 3 & 0 & 0 & 0 \\
0 & 0 & 0 & 4 / 3 & 0 & 0 \\
0 & 0 & 0 & 0 & 4 / 3 & 0 \\
0 & 0 & 0 & 0 & 0 & 2 / 3
\end{array}\right]\left[\begin{array}{l}
\partial T_{1} / \partial t \\
\partial T_{2} / \partial t \\
\partial T_{3} / \partial t \\
\partial T_{4} / \partial t \\
\partial T_{5} / \partial t \\
\partial T_{6} / \partial t
\end{array}\right]
$$

Substitution of $L=w / 5$ and $c=\rho C_{p}$ and $k_{y}=k$ reduces the assembly equation to the following set of linear first-order equations.

$$
\left\{\begin{array}{l}
\partial T_{1} / \partial t \\
\partial T_{2} / \partial t \\
\partial T_{3} / \partial t \\
\partial T_{4} / \partial t \\
\partial T_{5} / \partial t \\
\partial T_{6} / \partial t
\end{array}\right\}_{t}=\left[\begin{array}{cccccc}
0.15 & -0.15 & 0 & 0 & 0 & 0 \\
-.0 .075 & 0.15 & -0.075 & 0 & 0 & 0 \\
0 & -0.075 & 0.15 & -0.075 & 0 & 0 \\
0 & 0 & -0.075 & 0.15 & -0.075 & 0 \\
0 & 0 & 0 & -0.075 & 0.15 & -0.075 \\
0 & 0 & 0 & 0 & -0.15 & 0.15
\end{array}\right]\left\{\begin{array}{l}
T_{1} \\
T_{2} \\
T_{3} \\
T_{4} \\
T_{5} \\
T_{6}
\end{array}\right\}_{t}
$$

Equation (20) is the global heat diffusion equation from which the time history of the temperature distribution of the idealized system was studied.

\section{Results}

Equation (5.16) may not have a definite solution if the values of the objective function (temperature) at the borders and in the beginning of the process are not specified or known. Such specification is usually referred to as boundary condition and initial value respectively. Considering the particular case where the slab is initially at a temperature of $30^{\circ} \mathrm{C}$ (say) at time $\mathrm{t}=0$ while one of its ends is brought in contact with water reservoir at $\mathrm{T}_{\mathrm{w}}$ $=40^{\circ} \mathrm{C}$ at all times and the other side is subjected to convection to an environment at $\mathrm{T}_{\infty}=30^{\circ} \mathrm{C}$.

This poses a boundary value problem in $\mathrm{T}(\mathrm{t})$ whose solution would emerge from the substitution;

$$
T_{1}=T_{w}=40, T_{6}=T_{\infty}=30, \partial T_{1} /\left.\partial t\right|_{t}=\partial T_{6} /\left.\partial t\right|_{t}=0
$$

in equation (5.16) to obtain the following reduced equation

$$
\partial T_{2} / \partial t=3-0.075 T_{3}
$$




$$
\begin{array}{r}
\partial T_{3} / \partial t=-3+0.15 T_{3}-0.075 T_{4} \\
\partial T_{4} / \partial t=-0.075 T_{3}+0.15 T_{4}-2.25 \\
\partial T_{5} / \partial t=-0.075 T_{4}+2.25
\end{array}
$$

The resulting set of linear first order differential equation (21) is now solved subject to $T_{2}(0)=T_{3}(0)=$ $T_{4}(0)=T_{5}(0)=30$ as an initial value problem (IVP) with the expediency of MATLAB 'dsolve' command to arrive at the following exponential series:

$$
\begin{gathered}
\left.T_{2}\right|_{t}=220 / 9+5 / 9 e^{9 / 40 t}+5 e^{3 / 40 t}+1 / 4 t \\
\left.T_{3}\right|_{t}=110 / 3-5 / 3 e^{9 / 40 t}-5 e^{3 / 40 t} \\
\left.T_{3}\right|_{t}=100 / 3+5 / 3 e^{9 / 40 t}-5 e^{3 / 40 t} \\
\left.T_{4}\right|_{t}=230 / 9-5 / 9 e^{9 / 40 t}+5 e^{3 / 40 t}-1 / 4 t
\end{gathered}
$$

\section{Node 2 Time Response}

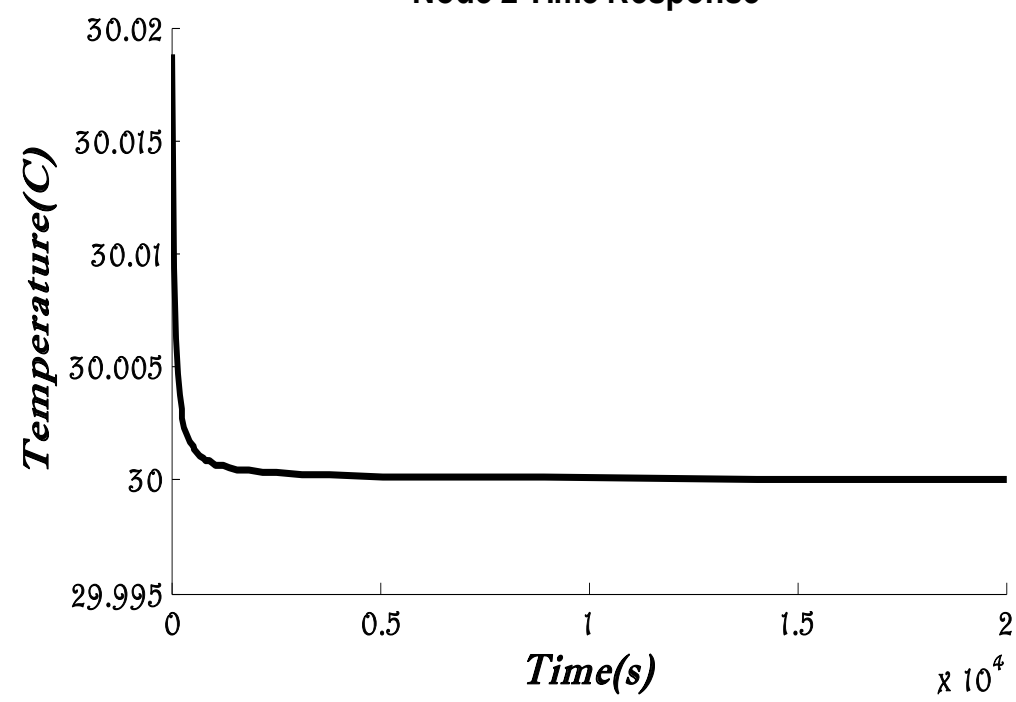

Figure 3(a) Node 2 time response

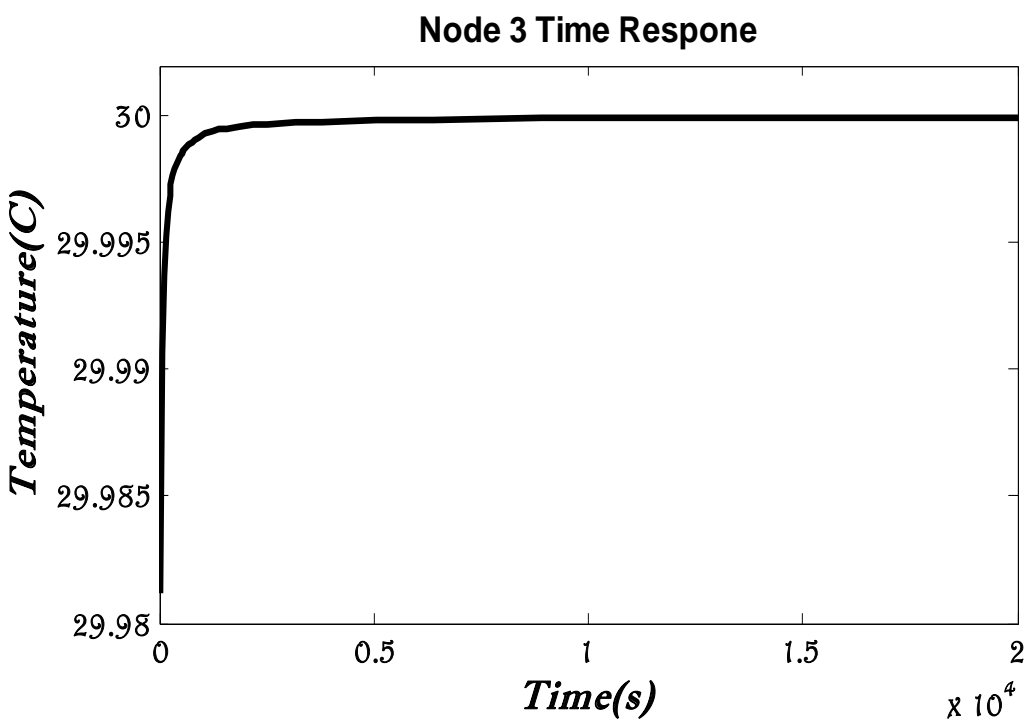

Figure 3(b) Node 3 time response 


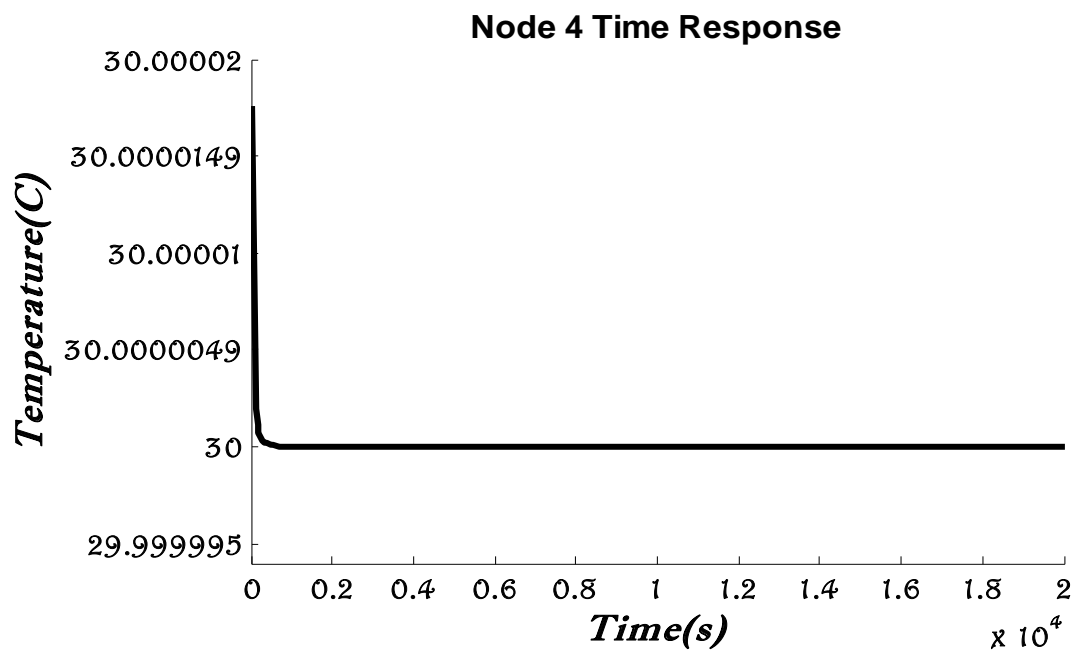

Figure 3(c) Node 4 time response

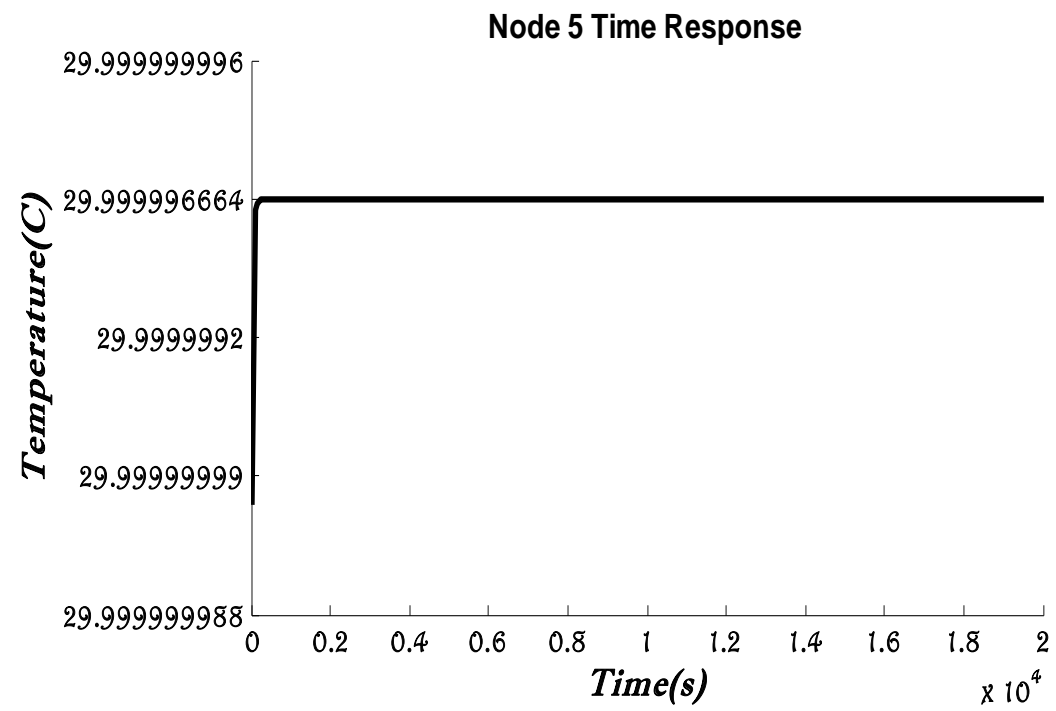

Figure 3(d) Node 5 time response

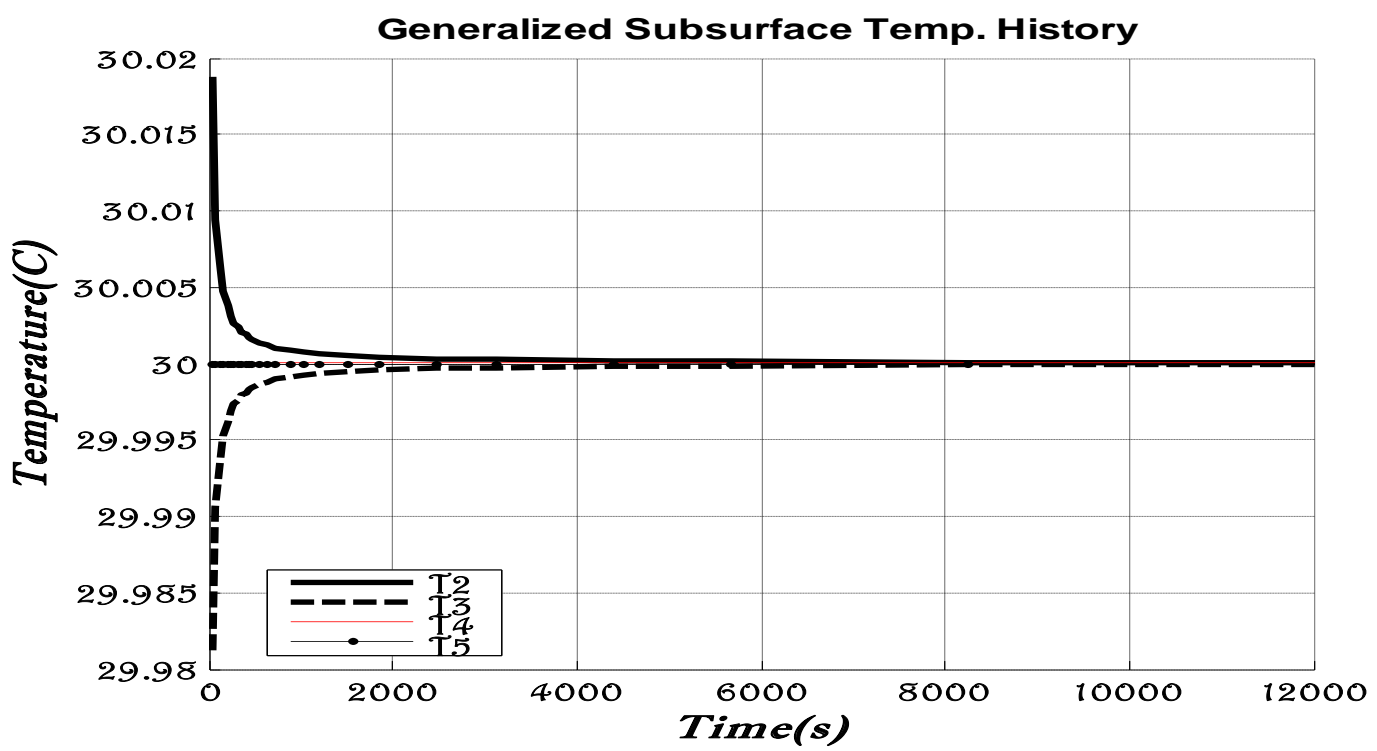

Figure 4: $\quad$ The generalize instantaneous subsurface temperature Profile 


\section{DISCUSSION}

The solution of the interior mesh points presented above with the boundary conditions and the initial values predict completely the time histories of temperature distribution across the isotherms overlaid through the thickness of the slab - figure $3 a-d$.

The result shows that during the conduction process when ' $t$ ' probably takes a value $>0$, the instantaneous nodal temperatures $T_{i}(t)$ assume peak and minimum values between adjacent nodes of a typical element. This implies that the diffusion of heat at the nodes closer to heat source for any given element is accompanied by a certain degree of heat addition at the adjacent node confirming the quasi-harmonic nature of heat flow across the region. Also, as the time approaches infinity the parameter everywhere converges progressively and exponentially to the value fixed at the low temperature reservoir.

This as well shows that the excess heat transmitted to the sub-surfaces due to the hot interface diffused rapidly into the low temperature reservoir through the oscillation of heat across the thickness of the slab. This characterize the conduction process and the associated surface convection, maintaining low temperature zones within the entire region over a long period of time. The low temperature profile maintained over time across the slab can also be attributed to the low thermal conductivity of the material observed in the model.

However, this result may not be the same for a case where the size of the heat source or sink (purported reservoir) is not large enough to sustain constant temperature at the boundaries (creating what may be regarded as a conditioned space). In such conditions, significant cooling effect is identified in space due to removal of sensible heat from the conditioned space in the form illustrated by figure $3 a$, but only to normalize on attaining the wet bulb temperature of the surrounding medium.

\section{Conclusion}

This research work has utilized the finite element model to study heat flows in a heat conducting slab. The result showed that during heat flows within and around the heat conducting slab, the instantaneous nodal temperatures $T_{i}(t)$ assume peak and minimum values between adjacent nodes of a typical element of the discretized slab. This implies that the diffusion of heat at the nodes closer to heat source for any given element is accompanied by a certain degree of heat addition at the adjacent node. This further verified the quasi-harmonic nature of heat flow across the region. Finally, as the time became infinite, the parameter everywhere converges progressively and exponentially towards the specified boundary condition at the lower temperature reservoir.

\section{References}

[1] Astley, R.J., (1992) Finite Element in Solids and Structures, Chapman and Hall Publishers, UK.

[2] Chadwick, P. (1976) Continuum Mechanics: Concise theory and problems, 2nd edition. Dover Publications, New York.

[3] Ihueze, C.C. and Ofochebe, S.M., Finite Design for Hydrodynamic Pressures on Immersed Moving Surfaces International Journal of Mechanics and Solids, ISBN 0973-1881 Volume 6, Number 2(2011), pp. 115-128 Research India Publications.

[4] Steven C. Chapra and Raymond P. Canale (1998) Numerical methods for engineers: with programming and software applications, 3rd edition. The McGraw - Hill Companies, Inc.

[5] Ziennkiewicz, O.C. and Cheung, Y.K. (1967) The Finite Element in Structures and Continuum Mechanics, McGrawHill Publishing Company Ltd. London pp. 166-168. 